\title{
The effect of proteinuria on relative mortality in Type 1 (insulin-dependent) diabetes mellitus
}

\author{
K. Borch-Johnsen ${ }^{1}$, P. K. Andersen ${ }^{2}$ and T. Deckert ${ }^{1}$ \\ ${ }^{1}$ Steno Memorial Hospital, Gentofte and ${ }^{2}$ Statistical Research Unit, University of Copenhagen, Copenhagen, Denmark
}

Summary. We followed 1, 134 patients with Type 1 (insulindependent) diabetes, diagnosed between 1933 and 1952, until 1982 or death or until their emigration. Their age at onset of diabetes was under 31 years. Information concerning the development of persistent proteinuria was sought in every case. In 104 cases, the data were either questionable or the patient could not be traced. Twenty-nine patients developed non-diabetic proteinuria. Among the remaining 1,001 patients, 406 developed persistent proteinuria $(350 \mathrm{died})$ and $595 \mathrm{did}$ not (166 died). The incidence of persistent proteinuria was highest among men; it decreased with increasing year of diabetes onset from 1933 to 1952, and decreased with increasing age at onset. The relative mortality was extremely high among patients with persistent proteinuria, increasing to a maximum of about 100 at age 35 years. Patients not developing proteinuria had a relatively constant low relative mortality of about 2 . The decreasing incidence of persistent proteinuria and the decreasing mortality with increasing calender year of diabetes onset resulted in a 50\% increase in life-expectancy among patients diagnosed in 1950 compared with patients diagnosed in 1935. In patients who developed persistent proteinuria, relative mortality was higher in women than men at all ages. In patients who did not develop proteinuria, relative mortality was similar in men and women after the age of 35 . Uraemia was the main cause of death in patients with persistent proteinuria, although cardiovascular deaths were more frequent than in patients without proteinuria. Thus, proteinuria is associated not only with death from uraemia but also from cardiovascular disease. It is concluded that the development of persistent proteinuria is a major life-threatening complication in patients with early-onset Type 1 diabetes. Patients who do not develop proteinuria have almost a normal life expectancy.

Key words: Persistent proteinuria, relative mortality, prognosis, Type 1 diabetes, diabetic complications, angiopathy, nephropathy
The substantially increased mortality from arterial diseases in subjects with diabetes mellitus has long been recognized $[1,2]$. It is also well-known that the reduced incidence of severe arterial disease in women is not found in diabetic patients [1,3]. These findings are, however, mostly based upon epidemiological observations in Type 2 (non-insulin-dependent) diabetic patients. Recent observations in patients with early onset Type 1 diabetes have demonstrated that relative mortality decreases with increasing age, indicating that mortality from arterial diseases in diabetic patients was less important than previously believed. These studies also demonstrated relative mortality to be identical in male and female diabetic patients, indicating that the usual protection of females was present $[4,5]$. This discrepancy between the mortality of Type 2 and Type 1 diabetic patients may have been due to heterogeneity of the subjects studied. In fact, relative mortality in insulin-dependent diabetes is highly variable, being much higher in patients who develop persistent proteinuria than in patients who do not [4]. Furthermore, the incidence of clinical nephropathy was found to be significantly higher in men compared with women with Type 1 diabetes [6], an observation which probably influences sex-dependent relative mortality. It has also been shown that patients with Type 2 diabetes with an increased urinary albumin excretion rate have an increased risk for cardiovascular death compared with patients with a normal albumin excretion rate, again indicating that mortality figures are influenced by proteinuria [7, 8]. These observations suggest the need for further study of relative mortality in insulin-dependent diabetic subjects. In this study, the relative mortality of a large number of early-onset Type 1 diabetic patients is analysed in relation to the occurrence of persistent proteinuria.

\section{Study population}

We studied all patients admitted to the Steno Memorial Hospital with early-onset, Type 1 diabetes (onset before age 31 years) and diagnosed between 1933 and 1952. The patients were referred between 1933 and 1975 for diabetic advice.

After the first admission to the Steno Memorial Hospital, $40 \%$ were followed there, while $60 \%$ were fol- 


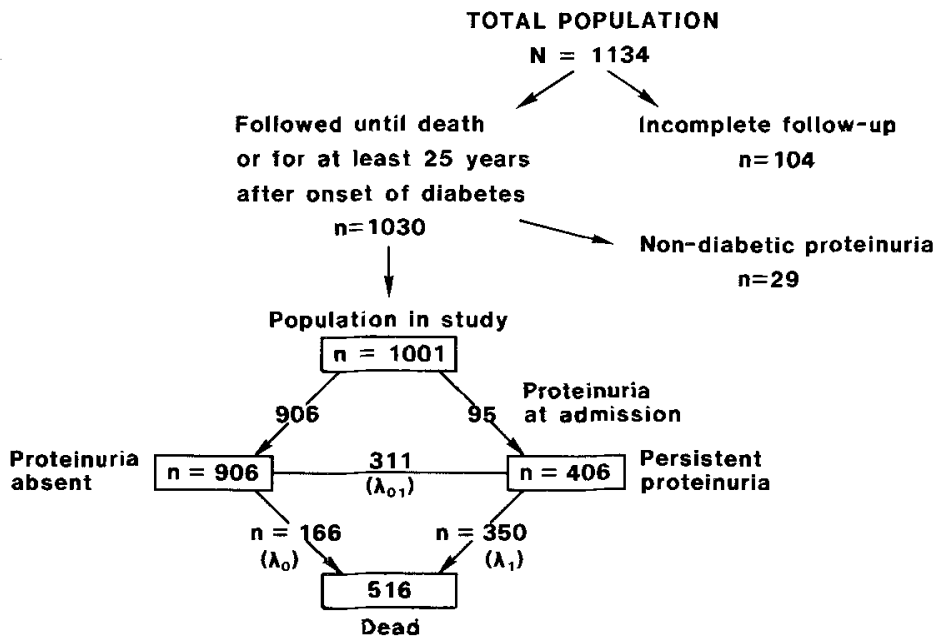

Fig. 1. The subdivision of the cohort of 1,134 insulin-dependent diabetic patients (see text for details). $\lambda_{0}=$ death intensity of patients without persistent proteinuria $\lambda_{01}=$ incidence of persistent proteinuria $\lambda_{1}=$ death intensity of patients with persistent proteinuria
Table 1. Clinical data of patients with a known date of onset of proteinuria and those with an unknown onset of proteinuria

\begin{tabular}{llll}
\hline & $\begin{array}{l}\text { Patients with } \\
\text { known onset } \\
\text { of proteinuria } \\
\end{array}$ & $\begin{array}{l}\text { Patients with } \\
\text { ynknown } \\
\text { onset of } \\
\text { proteinuria } \\
(n=291)\end{array}$ & $p$ \\
\hline Sex M:F & $178: 113$ & $80: 35$ & 0.11 \\
Year of diabetes onset & & & \\
$1933-1937$ & 81 & 36 & \\
$1938-1942$ & 81 & 36 & 0.55 \\
$1943-1947$ & 71 & 21 & \\
$1948-1952$ & 58 & 22 & \\
Age at onset (years) & & & \\
$0-10$ & 112 & 38 & \\
$11-20$ & 121 & 53 & \\
$21-30$ & 58 & 24 & \\
\hline
\end{tabular}

Significance according to $\chi^{2}$ test

lowed by general practitioners, possibly in collaboration with a local department of internal medicine. Records from the general practitioners and/or the department of internal medicine were requested in every patient. All patients were followed until January 1982, or until their emigration or death. The study population consisted of 1,134 patients (Fig.1) and was the same as previously described [6], although those who developed diabetes before 1933 were excluded because diabetes care was not properly organized and long-acting insulin preparations were not in use. From this population, 29 patients were excluded because persistent proteinuria developed from renal diseases other than diabetic nephropathy. Furthermore, in 104 patients, the development of proteinuria was questionable or the patients could not be followed for more than 25 years and were consequently excluded. Among the remaining 1,001 patients (556 men, 445 women), 595 did not develop persistent proteinuria $(>0.5 \mathrm{~g}$ protein $/ 24 \mathrm{~h}$ in at least four consecutive samples of 24 -h urine with intervals of at least 1 month) and 166 of these patients died during the observation period (before 1982). Four hundred and six patients either had persistent proteinuria at admission $(n=95)$ or developed persistent proteinuria during the observation period $(n=311)$. In these patients, 350 died during the observation period. In 291 patients, the onset of persistent proteinuria could be accurately determined to \pm 1 year, while precise information on the time of onset of persistent proteinuria was unknown in 115 patients.

In these 115 patients, we had information about the last time each patient was seen without proteinuria, and the first time proteinuria was observed. The male/female ratio, age at onset of diabetes, and year of diabetes onset did not differ between patients with a precisely defined onset of persistent proteinuria and patients in whom the year of onset of persistent proteinuria could not be exactly determined (Table 1). Using data from patients in whom the time of onset was known, the most probable time of onset of persistent proteinuria was estimated as the conditional mean given: sex and time of the last visit without proteinuria and the time of the first visit with proteinuria.

The main causes of death were taken from death certificates and/or hospital records. In several cases, multiple causes of death were present. If, however, the serum creatinine level was $>500 \mu \mathrm{mol} / \mathrm{l}$, uraemia was taken as the cause of death.

\section{Definitions used}

'Relative mortality' is defined as the observed number of deaths given the sex, age (a) and calendar-year (t) in the diabetic population, divided by the expected number of deaths given the sex, age (a) and calendar-year (t) based on the non-diabetic population.

'Absolute mortality' is defined as the percentage of a population with a given set of characteristics dying per year.

'Survival probability' is defined as the probability of being alive at age $a+x$, given the individual was alive at age $\mathrm{a}$. 
'Persistent proteinuria' is defined as a protein excretion $>0.5 \mathrm{~g} / 24 \mathrm{~h}$ in at least four consecutive urine samples with an interval of at least 1 month in patients without renal infection.

The following symbols are used; $\lambda_{0}=$ death intensity of patients not developing persistent proteinuria; $\lambda_{01}=$ intensity ( $=$ incidence) of persistent proteinuria; $\lambda_{1}=$ death intensity of patients with persistent proteinuria.

\section{Statistical model}

In the statistical analyses, separate models were devised for the three intensities shown in Figure 1: the death intensity $\lambda_{0}(\cdot)$ for patients without persistent proteinuria, the death intensity $\lambda_{1}(\cdot)$ for patients with persistent proteinuria, and the intensity (incidence) $\lambda_{01}(\cdot)$ of persistent proteinuria. As the present study covers a long period (1933-1981), considerable changes in the mortality in the general population took place, and, therefore, at the first stage, a modified Cox regression model for relative mortality was used for the analysis of the death intensities $\lambda_{0}(\cdot)$ and $\lambda_{1}(\cdot)[9]$.

Thus, the models are expressed as

$$
\begin{aligned}
& \lambda_{0}(\mathrm{a}, \mathrm{z})=\mu_{\mathrm{s}}(\mathrm{a}, \mathrm{t}) v_{0}(\mathrm{a}) \exp \left(\beta_{01} \mathrm{z}_{1}(\mathrm{a})+\ldots+\beta_{0 \mathrm{k}} \mathrm{z}_{\mathrm{k}}(\mathrm{a})\right) \\
& \lambda_{1}(\mathrm{a}, \mathrm{z})=\mu_{\mathrm{s}}(\mathrm{a}, \mathrm{t}) \nu_{1}(\mathrm{a}) \exp \left(\beta_{11} \mathrm{z}_{1}(\mathrm{a})+\ldots+\beta_{1 \mathrm{k}} \mathrm{z}_{\mathrm{k}}(\mathrm{a})\right)
\end{aligned}
$$

where $\lambda_{0}(\mathrm{a}, \mathrm{z})$ and $\lambda_{1}(\mathrm{a}, \mathrm{z})$ were the death intensities at age a for diabetic patients with covariates $z_{1}(a), \ldots, z_{k}(a)$ without and with persistent proteinuria, respectively. Furthermore, $\mu_{s}(a, t)$ was the death-intensity of the general population for a person of same sex and current age at calendar time $\mathrm{t}, \beta_{01}, \ldots, \beta_{0 \mathrm{k}}$ and $\beta_{11, \ldots,} \beta_{1 \mathrm{k}}$ were unknown regression-coefficients to be estimated $v_{0}(\mathrm{a})$ and $v_{1}(\mathrm{a})$ represent the age-specific baseline relative mortalities, i.a. the relative mortalities for patients of age $a$, in whom $z_{1}(a)=\ldots=z_{k}(a)=0$, in patients without or with persistent proteinuria, respectively.

The covariates included sex, age at diagnosis, calendar-year of diagnosis, and calendar time during followup. Analysing the mortality of patients with persistent proteinuria, we first studied relative mortality using the above model, and, second, used an ordinary Cox regression model for absolute mortality, i.e. without inclusion of the factor $\mu_{\mathrm{s}}(\mathrm{a}, \mathrm{t})$.

For analysis of the incidence of persistent proteinuria, a Cox regression model with time defined as duration of diabetes was used. Since information on sex was used in the prediction of the time of onset of persistent proteinuria for those patients where the exact year of onset was unknown, a Cox regression model stratified by sex was used in the analysis of the incidence of persistent proteinuria. Thus, separate intensities of persistent proteinuria were estimated for men and women and no statistical comparisons between the two were performed.
Table 2. Clinical characteristics of the patients studies according to the development of proteinuria

\begin{tabular}{llll}
\hline & $\begin{array}{l}\text { Patients who } \\
\text { did not devel- } \\
\text { op proteinuria } \\
(\mathrm{n}=595)\end{array}$ & $\begin{array}{l}\text { Patients who } \\
\text { develop pro- } \\
\text { teinuria } \\
(\mathrm{n}=406)\end{array}$ & $p$ \\
\hline Sex M:F & $298: 297$ & $258: 148$ & $<0.001$ \\
Year of diabetes onset & 131 & 117 & \\
$\begin{array}{l}\text { 1933-1937 } \\
1938-1942\end{array}$ & 149 & 117 & 0.002 \\
$1943-1947$ & 141 & 92 & \\
$1948-1952$ & 174 & 80 & \\
Age at onset (years) & & & \\
$0-10$ & 193 & 150 & 0.003 \\
$11-20$ & 225 & 174 & \\
$21-30$ & 177 & 82 & \\
\hline
\end{tabular}

Significance according to $\chi^{2}$ test

For each of the three separate models, the patients were included in the 'risk set' from entry into the study (i. e. first admission to the Steno Memorial Hospital).

The regression coefficients were estimated by maximizing the partial likelihood function [10], and the underlying intensities were estimated according to Ramlau-Hansen [11]. Hypotheses concerning the regression coefficients were tested by means of the partial likelihood ratio rest statistic $-2 \log \mathrm{Q}$ having an approximate $\chi^{2}$ distribution with the number of degrees of freedom equal to the difference between the number of regression coefficients outside and under the hypothesis tested. First the variables were entered in singlefactor analyses and only the significant variables were included in the multivariat Cox regression models.

From estimates of the intensities $\lambda_{0}(\cdot), \lambda_{1}(\cdot)$ and $\lambda_{01}(\cdot)$, it is possible to obtain estimates for the survival probability corresponding to any value of diabetes duration for an individual for a given sex, age at diagnosis and calendar year at diagnosis.

\section{Results}

Table 2 shows the clinical characteristics of patients developing and not developing persistent proteinuria.

\section{Incidence of persistent proteinuria}

Only patients without proteinuria at admission were included in the incidence study. Figure 2 shows the incidence of persistent proteinuria in the entire material. It can be seen that the incidence rose dramatically after 10 years of diabetes, reached a maximum after 13-18 years of diabetes, after which it decreased. No second peak of incidence was found. Women had a reduced incidence of persistent proteinuria compared with men. The difference between men and women was most pronounced at 10-30 years of diabetes, while no sex difference was found, for a duration of less than 10 years or 


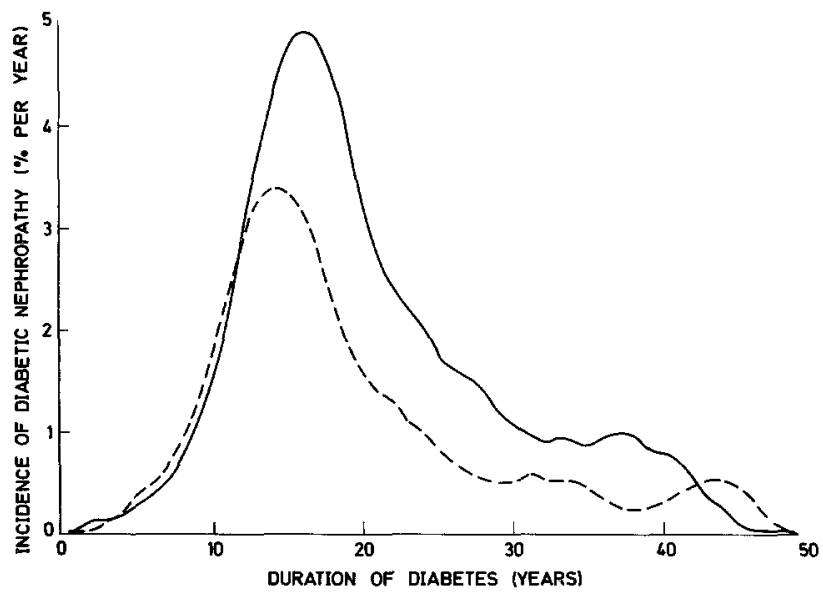

Fig. 2. The incidence of diabetic nephropathy (persistent proteinuria) as a function of diabetes duration. ---- women; - men

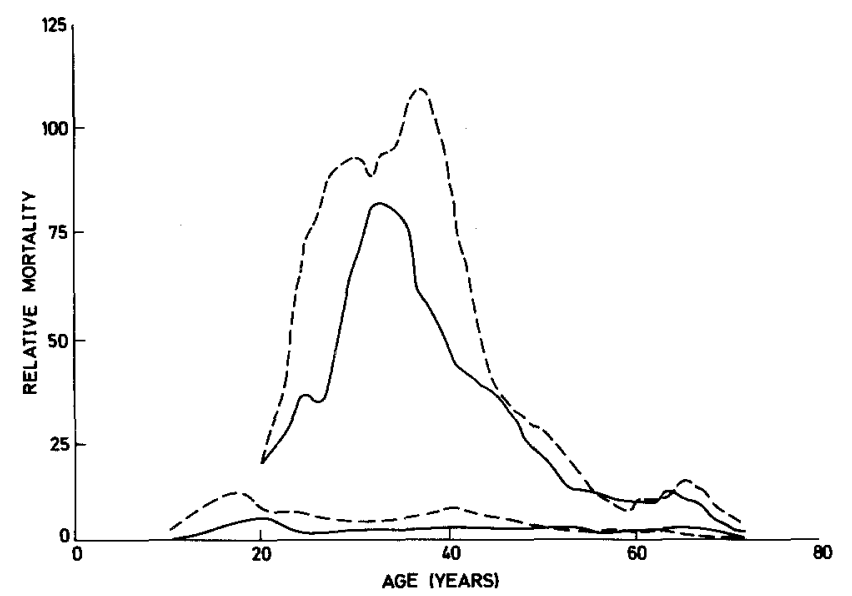

Fig. 3. The relative mortality among patients with persistent proteinuria (upper curves) and without proteinuria (lower curves) as a function of current age. ----- women; - men

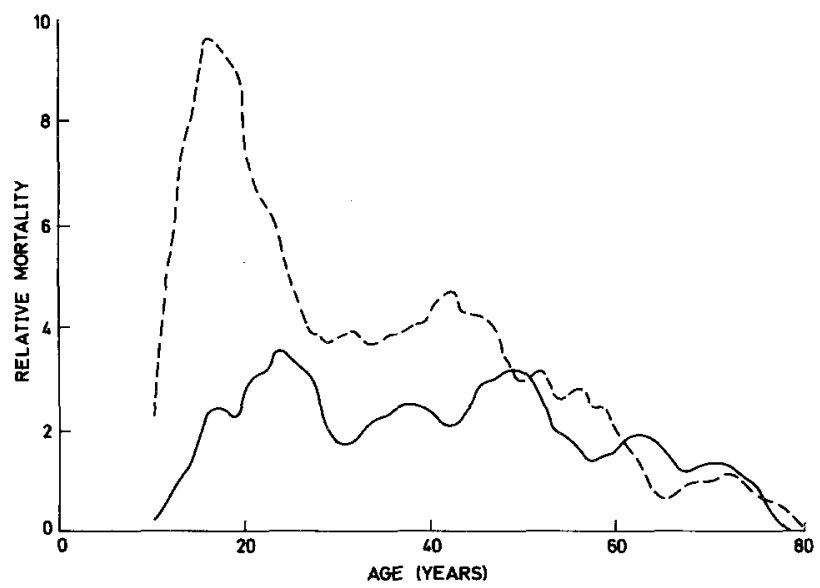

Fig.4. The relative mortality of patients without proteinuria as a function of current age. ---- women; - men

longer than 30 years. The incidence of persistent proteinuria decreased with increasing age at diagnosis $(-2 \log Q=6.56,1$ d.f., $p=0.01)$. When stratifying the age at diagnosis in 10-year intervals, the incidence could be considered constant from the ages of 1-20 years at diagnosis, but then it declined considerably in patients who were older at diagnosis $(-2 \log \mathrm{Q}=11.66,2 \mathrm{~d}$.f., $p=0.003$ ).

The incidence of persistent proteinuria was highest among patients diagnosed between 1933 and 1938, after which the incidence declined, the lowest incidence occurring in patients diagnosed between 1948 and 1952 $(-2 \log \mathrm{Q}=16.02,3$ d.f., $p=0.001)$. In the singlefactor analysis, a significant effect of the calendar year during follow up on the incidence of persistent proteinuria was found. The highest incidence was seen in the period 1951-1960, but when the effect of calendar year at diagnosis, age at diagnosis, and sex were included in the analysis, the effect of the calendar year during follow up on the incidence of persistent proteinuria was not significant $(2 \log \mathrm{Q}=6.48,3$ d.f., $p=0.09)$.

\section{Mortality}

A marked difference was found in relative mortality between patients with persistent proteinuria and those without proteinuria (Fig.3). Among patients with persistent proteinuria, a steep increase in relative mortality was found at the age of 20 years, with a maximal relative mortality of nearly 100 in the age range $30-40$ years (corresponding to a median duration of diabetes of 15-25 years). After this peak, a sharp decline in relative mortality was seen. Among patients not developing persistent proteinuria, the relative mortality was about two and remained relatively constant throughout life.

Among patients not developing persistent proteinuria, significantly higher relative mortality was found among women than among men $(-2 \log Q=6.16$, 1 d.f., $p<0.01$; Fig. 4). However, when stratifying age in the intervals 0-17 (childhood and puberty), 18-35 (child-bearing), 36-50 (pre-menopausal) and 50+ years (post-menopausal), a higher relative mortality among women was only seen in the first two age intervals.

A non-significant trend towards decreasing relative mortality with increasing age at diagnosis was found $(-2 \log \mathrm{Q}=3.38,1$ d.f., $p=0.07)$. When grouping the subjects according to 5-year intervals of onset of diabetes from 1933 to 1952, the relative mortality was highest among patients diagnosed from 1938 to 1942 , but it then decreased significantly $(-2 \log Q=13.04,3$ d.f., $p=0.005$ ). With time during the follow-up period as a continuous variable, no change was found during the follow-up period from 1933 to $1981(-2 \log Q=2.10$, 3 d.f., $p=0.55$ ), i.e. patients not developing persistent proteinuria had the same relative change in mortality during the observation period as the non-diabetic population.

In patients with persistent proteinuria, relative mortality was also significantly higher among women than among men. However, relative mortality was much higher than in patients not developing persistent proteinuria, reaching a maximum of 110 among women 


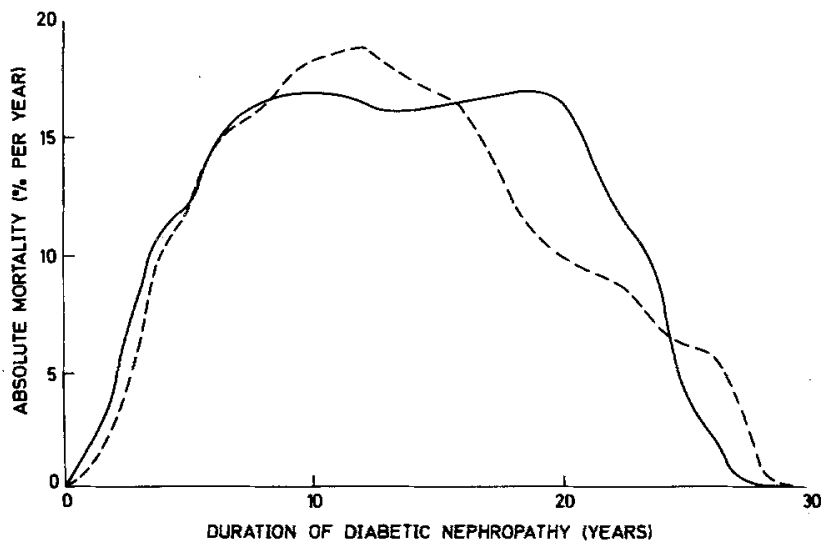

Fig.5. Absolute mortality among patients with persistent proteinuria as a function of duration of proteinuria. ---- women; - men

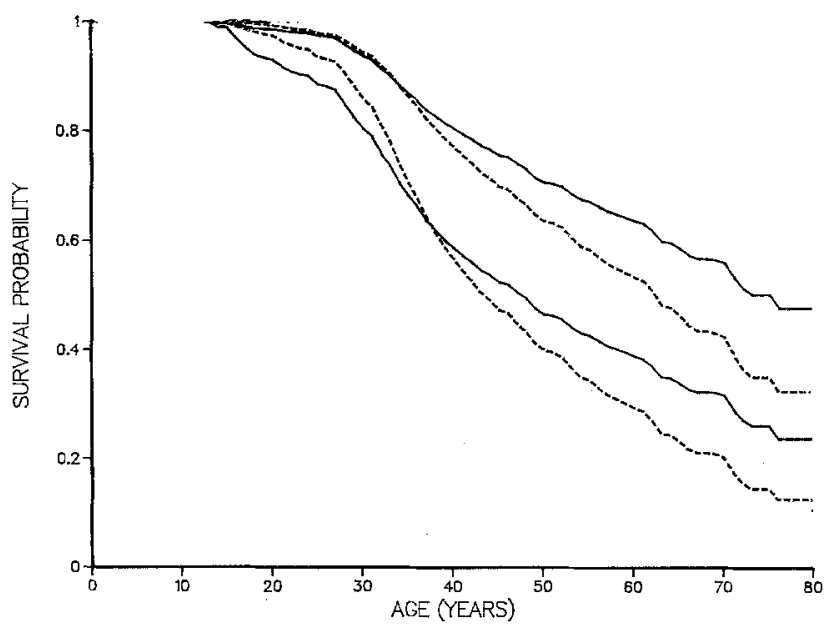

Fig.6. Estimated survival probability of Type 1 diabetic patients; age at diagnosis 10 years, - women -.-- men, Time of diagnosis: upper curves 1950, lower curves 1935

aged $36-38$ years, and 80 among men aged $34-36$ years (Fig. 3).

Since median survival after onset of proteinuria is only 7-8 years [6], and since the mortality in the nondiabetic population is higher among men than among women up to 85 years of age, we used absolute mortality in the analyses of prognostic factors of patients with persistent proteinuria. The mortality in patients with persistent proteinuria (Fig.5) shows that mortality was low during the first 3 years of proteinuria, after which it increased to a constant level of $17 \%$ per year, 10-20 years after the onset of proteinuria. Patients with proteinuria for more than 20 years demonstrated a lower mortality. After the onset of proteinuria, mortality was the same in men and women $(-2 \log Q=0.26$, 1 d.f., $p=0.61$ ). This means that the difference in the relative mortality between men and women was due to differences in mortality in the non-diabetic population and not due to different influences of persistent proteinuria in men and women. The highest mortality was found among patients with onset of diabetes between 1938 -1942. After this, the mortality decreased significantly $(-2 \log \mathrm{Q}=11.37,3$ d.f., $p=0.01)$. The age at onset of diabetes showed no effect on the mortality of dia-
Table 3. Cause of death among patients with persistent proteinuria, patients without proteinuria and in a reference population ${ }^{a}$

\begin{tabular}{llll}
\hline & $\begin{array}{l}\text { Patients with } \\
\text { persistent pro- } \\
\text { teinuria }\end{array}$ & $\begin{array}{l}\text { Patients with- } \\
\text { out protein- } \\
\text { uria }\end{array}$ & $\begin{array}{l}\text { Reference }^{\mathrm{a}} \\
\text { population } \\
(1972) \text { aged }\end{array}$ \\
& $(\%)(n=350)$ & $\begin{array}{l}\text { 35 -59 years } \\
(\%)(n=166)(n=7279)\end{array}$ \\
\hline Uraemia & 66 & - & 0.3 \\
Cardiovascular & 23 & 45 & 25.3 \\
diseases & & & \\
Hypoglycaemia & 1 & 4 & 0 \\
Ketoacidosis & 1 & 8 & 0 \\
Diabetes mellitus & 0.3 & 0 & 0 \\
Infections & 4 & 20 & 4.1 \\
Neoplasia & 0.3 & 7 & 34.9 \\
Suicide & 0.3 & 2 & 8.5 \\
Others and & 5 & 15 & 26.9 \\
$\quad$ unknown & & & \\
\hline
\end{tabular}

a Danmarks Statistik, Statistical Yearbook, Denmark 1972

betic patients with proteinuria $(-2 \log \mathrm{Q}=0.011,1 \mathrm{~d}$.f., $p=0.92$ ). Year at onset of persistent proteinuria showed a significant effect on mortality. However, when year of diagnosis, diabetes duration at onset of persistent proteinuria and duration of persistent proteinuria were included in the analysis, no significant change in mortality during the observation period was found $(-2 \log \mathrm{Q}=6.23,3$ d.f., $p=0.10)$.

Early onset of persistent proteinuria was a more serious event than late onset. When the interval between diabetes onset and onset of proteinuria was stratified into the intervals: $0-10$ years, $11-15$ years, $16-20$ years and $21+$ years, a highly significant effect of mortality was found $(-2 \log Q=31.2,3$ d.f., $p<0.0001)$, indicating that patients developing persistent proteinuria after more than 15 years of diabetes had a better prognosis than patients developing it earlier.

If sex, year of onset of diabetes, year during followup, incidence of persistent proteinuria and changes in mortality in the non-diabetic population were taken into consideration, the survival probability of all Type 1 diabetic patients with fixed age at onset, and fixed year of onset could be estimated (Fig. 6). Figure 6 shows that median survival was $50 \%$ longer if patients who were 10 years of age at diagnosis were diagnosed in $\mathbf{1 9 5 0}$ compared with 1935.

\section{Cause of death}

Eighty-six percent of patients who developed persistent proteinuria died, whereas only $28 \%$ of the others died before 1982. The causes of death are seen in Table 3. Since the deaths occurred during the period 1933-1982, it was impossible to compare causes of death in our material with those in the non-diabetic population. Therefore, causes of death in a recent Danish population of comparable age are given. 
A comparison of the causes of death between the two diabetic populations demonstrated differences in death from uraemia, cardiovascular diseases (ischaemic heart and cerebrovascular disease), ketoacidosis, infectious diseases, neoplasm and others. Eighty out of the 406 patients in the group with proteinuria died from cardiovascular events (these patients with proteinuria were followed for a total of 3,295 years), whereas 75 out of the 595 patients without proteinuria died from these causes (these patients without proteinuria were followed for a total number of 19,443 years), indicating that the mortality from cardiovascular events was higher among patients with persistent proteinuria than in patients without persistent proteinuria.

\section{Discussion}

The present study material consists of patients with Type 1 diabetes diagnosed between 1933 and 1952, and under 31 years of age. It has been shown previously that this population is well characterized and representative of diabetic patients of a similar age in Denmark with respect to age at diagnosis and relative mortality $[5,6]$. As only patients without proteinuria at admission were included in the incidence study, it is unlikely that the effects of age at diagnosis and year of onset of diabetes we observed were due to changes in patient admission to the hospital.

The incidence of persistent proteinuria in this study was very similar to that found previously [6], although patients with diagnosis before 1933 were excluded here, and the precise time of onset of proteinuria was unknown in 115 patients. However, the second incidence peak after 32 years of diabetes found in the previous study [6], was not seen, probably because many longterm survivors among patients diagnosed before 1933 were excluded. As shown previously [6, 12], the incidence of persistent proteinuria was higher among men than among women. The reason for this sex bias is still unknown. It is, however, also present in other renal diseases, such as glomerulonephritis [13]. A new observation here is that a young age at diagnosis is associated with an increased incidence of persistent proteinuria. This probably explains our previous findings [5] of highest relative mortality among patients diagnosed at $0-10$ years of age. The cause of this difference remains unexplained, but may be attributed to the deleterious effect of poor metabolic control compounded by the difficulties in treating teenagers. The decreasing incidence of persistent proteinuria with increasing year of diagnosis probably explains the previously reported decreasing relative mortality with increasing year of diagnosis $[4,5]$. Indeed, life expectancy for patients diagnosed aged 10 was much longer in 1950 than in 1935. The cause of this decreasing incidence of persistent proteinuria remains unknown, but it is likely to reflect improvements in the management of diabetes from 1933 onwards.
In a recent study, we demonstrated that relative mortality increased with increasing duration of diabetes to a maximum at $15-25$ years, after which it declined considerably, and that relative mortality was independent of sex $[4,5]$. However, we were unable to analyse the impact of proteinuria on prognosis in this study as data concerning development of persistent proteinuria were not available in approximately $50 \%$ of the patients.

The present study reveals that high relative mortality is mainly related to the development of persistent proteinuria. While relative mortality among patients not developing persistent proteinuria was virtually constant at about twice the mortality in the reference population, relative mortality among patients developing persistent proteinuria reached a maximum of $80 \mathrm{in}$ men and 110 in women, the difference between the sexes being significant. This difference was, however, due to the fact that mortality rates in Denmark, as in most other countries, are higher among men than among women in all age groups up to 85 years of age (Danmarks Statistik, Statistical Yearbook). Absolute mortality was similar between the sexes in patients with persistent proteinuria, indicating that the usual protection of women from severe vascular complications is abolished in this group of patients. The lack of sex difference in relative mortality, which was found previously [5] among unselected groups of Type 1 diabetic patients (including patients with and without proteinuria), results from a combination of the higher incidence of persistent proteinuria among men, and the higher relative mortality in diabetic women with proteinuria.

We also observed that mortality in the group of patients with persistent proteinuria decreased with increasing year of diagnosis. This means that the median survival after the onset of proteinuria increased with increasing year of onset of diabetes. It has been shown that intensive anti-hypertensive treatment can reduce the decline of renal function in patients with diabetic nephropathy. However, this could not explain the present finding, since intensive anti-hypertensive treatment was uncommon before 1980 .

Recent studies have shown that increased urinary albumin excretion rate is a predictor, not only of clinical diabetic nephropathy, but also of cardiovascular disease $[7,8]$. This is also the case in the present study. The number of cardiovascular deaths in patients who developed proteinuria was higher than in those who did not develop proteinuria, despite a smaller number of patients, and a shorter observation period in the former group. The cause of increased cardiovascular mortality in diabetic patients with increased urinary albumin excretion rates remains unknown. However, it has been shown previously that Type 1 diabetic patients with increased urinary albumin excretion rates have increased blood pressure [14], higher cigarette consumption [15] enhanced platelet adhesion [16], higher blood viscosity [17], increased transcapillary escape rate [18, FeldtRasmussen, unpublished observations], and elevated 
plasma fibrinogen concentrations [19], compared with diabetic patients of similar duration without complications. Thus, these multiple, complex cardiovascular risk factors may explain the substantial increase in cardiovascular mortality in subjects with persistent proteinuria.

In patients not developing persistent proteinuria, the main causes of death during the first 20 years of diabetes were infections, such as tuberculosis and ketoacidosis. Higher relative mortality among women in this early period of diabetes indicates that ketoacidosis is a special risk factor for young female patients. Indeed, 9 out of 21 patients, dying before the age of 25 years, died from ketoacidosis (6 women and 3 men). Among patients dying after 20 years of diabetes, the main cause of death was cardiovascular disease. We do not know whether the incidence of cardiovascular death in this group of diabetic subjects was higher than in the nondiabetic population, since comparable data are not available. Nevertheless, over-representation of cardiovascular deaths was small, and after age 35 years, when cardiovascular disease was the main cause of death, a sex difference in relative mortality could not be seen in this group of patients.

The observation that the majority of Type 1 earlyonset diabetic patients who do not develop persistent proteinuria have an almost normal life expectancy was unexpected. This, together with the fact that capillary basement-membrane thickening, microaneurysms in the retina, and accumulation of basement membranelike material in the mesangium of the glomeruli can be seen in nearly all patients with long-term diabetes without severe complications, makes it possible to speak of a benign vasculopathy in such cases. High relative mortality in patients who did develop persistent proteinuria, the increased risk of cardiovascular death, the high prevalence of cardiovascular risk factors, and severe vascular renal and retinal changes seen in most of these patients make it possible to speak of 'malignant vasculopathy' in these patients, Such diabetic patients are characterized by a breakdown of the integrity of the vascular wall, not only in capillaries of the glomerular tuft, but also in the retina, muscles [20] and the intima of large arteries $[21,22]$. This 'malignant vasculopathy' remains a great challenge for diabetologists today.

In conclusion, our study shows that high relative mortality in patients with Type 1 diabetes is mainly restricted to those who develop persistent proteinuria. Decreasing relative mortality among patients diagnosed after 1933 was primarily due to the decreasing incidence of persistent proteinuria, resulting in an increase in life expectancy.

\footnotetext{
Acknowledgements. This study was supported by a grant from The Danish Diabetic Association (Diabetesforeningen). We are grateful for the assistance of Mrs. V. Rosenkrantz, National Board of Health, department $B$ and for her help with the death certificates. The assistance from General Practitioners and Departments of Internal Medicine, who provided us with information about the patients, is highly appreciated. The secretarial assistance of Mrs. J. Hvalsøe Olsen and Mrs. L. Zilstorff is also gratefully acknowledged.
}

\section{References}

1. Kannel WB, McGee DL (1979) Diabetes and glucose tolerance as risk factors for cardiovascular disease: the Framingham study. Diabetes Care 1: 120-126

2. Jarrett RJ, McCartney P, Keen H (1982) The Bedford Survey: tenyear mortality rates in newly diagnosed diabetics and normoglycaemic controls and risk indices for coronary heart disease in borderline diabetics. Diabetologia 22: 79-84

3. Jarrett RJ, Keen H, Chakrabarti R (1982) Diabetes hyperglycaemia and arterial disease. In: Keen $\mathrm{H}_{3}$ Jarrett $\mathrm{RJ}$ (eds) Complications of diabetes, 2nd edn. Arnold, London, pp 179-184

4. Deckert T, Poulsen JE, Larsen M (1979) The prognosis of insulindependent diabetes mellitus and the importance of supervision. Acta Med Scand (Suppl) 624: 48-53

5. Green A, Borch-Johnsen K, Kragh Andersen P, Hougaard P, Keiding N, Kreiner S, Deckert T (1985) Relative mortality of Type 1 (insulin-dependent) diabetes in Denmark 1933-1981. Diabetologia (in press)

6. Andersen AR, Christiansen JS, Andersen JK, Kreiner S, Deckert T (1983) Diabetic nephropathy in Type 1 (insulin-dependent) diabetes: an epidemiological study. Diabetologia 25: 496-501

7. Mogensen CE (1984) Microalbuminuria predicts clinical proteinuria and early mortality in maturity-onset diabetes. N Engl J Med $6: 356-360$

8. Jarrett RJ, Viberti GC, Argyropoulos A, Hill RD, Mahmud U, Murrells TJ (1984) Microalbuminuria predicts mortality in noninsulin-dependent diabetes. Diabetic Med 1: 17-19

9. Andersen PK (1984) A Cox regression model for the excess mortality in long-term follow-up studies. In: Proceedings from the XIIth International Biometric Conference, Tokyo, Japan, pp 157-162

10. Kalbfleisch JD, Prentice RL (1980) The statistical analysis of failure time data. Wiley, New York

11. Ramlau-Hansen $H$ (1983) Smoothing counting process intensities my means of kcrnel functions. Ann Statist 11: 453-466

12. Krolewski AS, Warram JH, Christlieb A, Busick EJ, Kahn CR (1985) The changing natural history of nephropathy in type 1 diabetes. Am J Med 78: 785-794.

13. Finn $R$, Morner $D$ (1979) Aetiological implications of sexratio in glomerulonephritis. Lancet 2: 1194

14. Mathiesen ER, Oxenbøll B, Johansen K, Svendsen PAa, Deckert $T$ (1984) Incipient nephropathy in Type I (insulin-dependent) diabetes. Diabetologia 26: 406-410

15. Telmer S, Christiansen JS, Andersen AR, Nerup J, Deckert T (1984) Smoking habits and prevalence of clinical diabetic microangiopathy in insulin-dependent diabetics. Acta Med Scand 215: 63-68

16. Valdorf-Hansen $F(1967)$ Nogle undersøgelser over trombocyt- og koagulationsforhold hos diabetikere (English summary) Thesis. University of Copenhagen

17. Skovborg F (1974) Blood viscosity in normal and diabetic subjects. A clinical-hemorrheological study. Thesis. FAD's Forlag, Copenhagen

18. O'Hare JA, Ferriss JB, Twomey B, O'Sullivan DJ (1983) Poor metabolic control, hypertension and microangiopathy independently increase the transcapillary escape rate of albumin in diabetes. Diabetologia 25: 260-263

19. Valdorf-Hansen F (1967) Coagulability in diabctics. Acta Mcd Scand (Suppl) 476: 147-157

20. Trap-Jensen J (1971) Permeability of small vessels in diabetes. Acta Diabet Lat 8 (Suppl): $192-200$

21. Stender $S$ (1982) The in vivo transfer of free and esterified cholesterol from plasma into the arterial wall of hypercholesterolemic rabbits. Scand J Clin Lab Invest 42 (Suppl) 161: 43-52

22. Ledet T, Gøtzsche O, Heickendorff $L(1984)$ The pathology of diabetic cardiopathy: pathogenetic reflections. In: Janet J (ed) Diabetes and heart disease. Elsevier Science Publications, Amsterdam, pp 25-46

Dr. Knut Borch-Johnsen

Steno Memorial Hospital

Niels Steensensvej 2

DK-2820 Gentofte

Denmark 